\title{
Low-energy electron diffraction study of the thermal expansion of $\operatorname{Ag}(111)$
}

\author{
E. A. Soares ${ }^{1}$, G. S. Leatherman ${ }^{2}$, R. D. Diehl ${ }^{2, *}$ and M. A. Van Hove H,3 $^{1,3}$ \\ ${ }^{1}$ Materials Sciences Division and Advanced Light Source, Lawrence Berkeley Laboratory, \\ Berkeley, CA 94720, USA \\ ${ }^{2}$ Physics Department, Penn State University, University Park, PA 16802, USA \\ ${ }^{3}$ Physics Department, University of California, Davis, CA 95616, USA
}

\begin{abstract}
The temperature dependence of the first three interlayer distances of the $\operatorname{Ag}(111)$ surface was studied by low-energy electron diffraction (LEED) over the temperature range $128 \mathrm{~K}$ to $723 \mathrm{~K}$. The first three interlayer spacings and the effective Debye temperatures were extracted from the LEED analysis. At the lowest temperature, the first two interlayer spacings are slightly $(0.5 \%)$ contracted. All three interlayer spacings increase with temperature, finally reaching expansions relative to the bulk of about $0.8 \%$ at the highest temperature studied. The effective surface Debye temperature is lowest for the outermost layer, increasing toward the bulk value for successive layers.
\end{abstract}

\section{Keywords}

low-energy electron diffraction (LEED), thermal expansion, silver

* Corresponding author: 104 Davey, Penn State University, University Park, PA 16802 (814)865-9251,(814)865-3604 (FAX); email: rdd2@psu.edu. 


\section{Introduction}

When a surface is produced, the bulk 3D periodicity is broken in one direction, reducing the symmetry of the surface. The surface atoms experience forces that are rather different from those exerted on atoms inside the crystal. Consequently, surfaces may be expected to exhibit different, perhaps anharmonic behavior, compared to the bulk. Anharmonicity should be observed in quantities such as the surface thermal expansion, thermal vibrations and the phonon distribution. Indeed, enhanced anomalous effects have been reported on many surfaces, such as $\mathrm{Pb}(110)$ [1-18].

There has been a recent interest in the thermal expansion of the $\operatorname{Ag}(111)$ surface, largely due to the anomalous thermal expansion behavior observed in medium-energy ion scattering (MEIS) experiments on that surface [1]. In that work, the structure of the $\operatorname{Ag}(111)$ surface was studied as a function of the temperature and it was found that the first interlayer spacing is contracted by $2.5 \%$ relative to bulk at temperatures below $670 \mathrm{~K}$, but at higher temperatures this interlayer spacing increases in a non-linear manner. At $1150 \mathrm{~K}$, $80 \mathrm{~K}$ below the bulk melting point, it is expanded $10 \%$ relative to bulk. A significant increase of the surface vibration amplitudes was also observed.

The first theoretical attempt to explain the observed contraction/expansion transition was performed by Lewis [7] using an embedded-atom-method (EAM) molecular dynamics (MD) simulation. In that study, seven different temperatures were investigated in the range

of 200-1100 K. The results of that calculation indicated a small contraction of the 
outermost surface interlayer spacing at $200 \mathrm{~K}$ and an essentially bulk-like expansion up to $1100 \mathrm{~K}$. Although the mean-square vibrational amplitudes agreed quite well with the MEIS results, the thermal expansion behavior was quite different.

Contrary to this EAM-MD study, a later density-functional theory (DFT) study of this surface produced thermal expansion behavior similar to the MEIS experiment [8]. The changes in the first $\operatorname{Ag}(111)$ interlayer spacing $\left(d_{12}\right)$ as a function of temperature were evaluated by minimizing the Helmholtz free energy of the system with respect to $d_{12}$. The static energies and the phonon frequencies were calculated at $\mathrm{T}=0 \mathrm{~K}$ using DFT theory within the local density approximation (LDA). The quantities obtained at $\mathrm{T}=0 \mathrm{~K}$ were then extended to finite temperatures using a simplified quasiharmonic approximation (QHA), where the vibrational free energy was evaluated considering only three representative modes, in all of which the topmost layer moves as a whole. The results from that calculation showed that, at low temperatures, the $\operatorname{Ag}(111)$ top layer is contracted by about $1.7 \%$ and at $\mathrm{T}=1040 \mathrm{~K}$ an expansion of $15 \%$ was observed in reasonable agreement with the experimental MEIS results. The interpretation of this "anomalous" expansion of the surface was attributed to the softening of parallel vibrational modes of the surface atoms rather than to the anharmonicity of the perpendicular modes, which was proposed to explain earlier observations of anomalous surface expansion on other surfaces $[2,3,5]$.

A later EAM-MD study by a different group essentially agreed with the first EAM-MD study cited above, indicating a surface contraction over the whole temperature range up to 
$1100 \mathrm{~K}$ and no anomalous expansion [9, 19]. This study went on to point out that the earlier DFT study did not include the full dynamical behavior of the system, and it was demonstrated that failing to include the full dynamics can give erroneous results for the thermal behavior of the surface. With this inducement, another DFT calculation, which included a more complete description of the dynamics but still within the quasiharmonic approximation [10] produced a somewhat more moderate thermal expansion of the surface, but the same driving mechanism for the thermal expansion.

Most of the experimental studies of surface thermal expansion have used MEIS as the experimental technique and only two, for $\mathrm{Be}(0001)$ [13] and very recently for $\mathrm{Cu}(111)$ [20], have employed a full dynamical LEED analysis to determine the surface geometries. There could be differences in the results of LEED and MEIS studies on surfaces at high temperatures where they may become rough. A comparison of the results of the two techniques may shed some light on the nature of the surface at elevated temperatures. In this paper we present a quantitative LEED analysis of the temperature dependence of the top three layers of the $\operatorname{Ag}(111)$.

\section{Experimental and Calculational Procedures}

The preparation of the $\operatorname{Ag}(111)$ surface has been described before [21]. The LEED experiments were carried out using a low-current LEED system which has been described earlier [22]. The LEED data were acquired using a charge-coupled device video camera with is interfaced to a personal computer via a Data Translation frame grabber and 
processor boards. The $\mathrm{I}(\mathrm{E})$ curves were measured directly from the LEED pattern for sample temperatures ranging from $128 \mathrm{~K}$ to $723 \mathrm{~K}$. The $\mathrm{I}(\mathrm{E})$ curves for the symmetricallyequivalent beams were averaged.

The calculation procedures were similar to those carried out in an earlier study of $\operatorname{Ag}(111)$ [23]. The full dynamical LEED calculations were performed using the LEEDFIT code [24-28]. In addition to the automatic optimization of the structural parameters, this code allows optimization of non-structural parameters such as the Debye temperature and the atomic vibrational amplitudes for each atom in each layer. A set of 10 phase shifts were calculated using the Barbieri/Van Hove phase shift package [29] and a muffin-tin radius of $1.44 \AA$. The three outermost layers were treated as being surface layers with potentially different structural parameters to those of the underlying bulk. The lattice parameter of the underlying bulk structure was calculated using the room temperature lattice constant [30] and the bulk thermal expansion coefficient. The imaginary part of the inner potential was optimized to $4 \mathrm{eV}$. The level of agreement between the experiment and the calculations was measured using the Pendry $R_{P}$ [31] and $R_{D E}$ [25]. The structural parameters were analyzed using both the grid method and an automatic optimization in order to obtain the best-fit structural model for the $\operatorname{Ag}(111)$ surface. The non-structural parameters, such as the Debye temperature and thermal vibrational amplitudes, were then optimized for the best-fit structural models in the final stage of refinement. The errors quoted reflect the statistical precision in the fitting. 


\section{Results}

Figure 1 shows the experimental curves measured as a function of temperature, along with the best-fit calculated curves. At the lowest temperature, 5 inequivalent beams were measured and the dataset has a total energy range $\left(\Delta \mathrm{E}_{\mathrm{T}}\right)$ of $1352 \mathrm{eV}$. As the temperature was increased, the energy range over which useable data could be acquired was reduced due to the increase in diffuse scattering. The dataset at $723 \mathrm{~K}$ had two beams with a total energy range of $204 \mathrm{eV}$. Figure 1 and the values of the R factors presented in Table 1 demonstrate that a good agreement between theory and experiment was obtained for all temperatures.

The interlayer spacings $\left(\mathrm{d}_{12}, \mathrm{~d}_{23}\right.$ and $\left.\mathrm{d}_{34}\right)$ and the Debye temperatures $\left(\theta_{\mathrm{D}}{ }^{1}, \theta_{\mathrm{D}}{ }^{2}\right.$ and $\left.\theta_{\mathrm{D}}{ }^{3}\right)$ determined from this analysis are given in Table 1 and are shown graphically in Figure 2. At the lowest temperature, $128 \mathrm{~K}$, the first interlayer spacing is contracted by $0.5 \%$, the second interlayer spacing is contracted by $0.4 \%$ and the third interlayer spacing is the same as the bulk interlayer spacing. As the temperature is increased, the interlayer spacings increase. At the highest temperature $(723 \mathrm{~K})$ each of these interlayer spacings is expanded by $0.8 \%$ relative to the bulk. This expansion, if assumed to have a linear dependence over this temperature range, corresponds to a thermal expansion coefficient of $22.0 \times 10^{-6} \mathrm{~K}^{-1}$ compared to the bulk value of $18.9 \times 10^{-6} \mathrm{~K}^{-1}$. Figure $2 \mathrm{~b}$ shows the Debye temperatures determined from the LEED calculations. These were essentially constant over the temperature range measured, to within the precision of the measurements. The average Debye temperatures from this analysis were $165 \pm 8 \mathrm{~K}, 199 \pm 9 \mathrm{~K}$, and $212 \pm 9 \mathrm{~K}$, 
respectively, for the first three layers. Published values of the Debye temperature of bulk $\mathrm{Ag}$ are in the range $215-225 \mathrm{~K}[30,32](225 \mathrm{~K}$ was the value used in this work). Since the Debye temperature is derived assuming a harmonic model for the thermal vibrations, the essentially constant behavior obtained here might be an indication that anharmonic effects are small in this temperature range.

Figure 3 compares the first interlayer spacing determined in this study to those obtained by the MEIS, DFT and EAM-MD studies described earlier. At low temperatures, the surface is slightly contracted, in agreement with the other studies. The magnitude of the contraction determined in this study is somewhat less than that found in either the DFT or EAM calculation, but is significantly smaller than that found in the MEIS experiment. As the temperature increases, the LEED result closely parallels that of the DFT calculation, while departing somewhat from the EAM-MD result, which remains essentially constant in the temperature range studied here. The MEIS result also changes very little over the range studied here, but begins to increase dramatically at a temperature of about $750 \mathrm{~K}$. The point where the LEED result crosses the no-contraction line is in agreement with an earlier LEED study of this surface using data collected at room temperature [23].

\section{Discussion and Conclusion}

Figure 4 shows experimental data for the reduced-temperature dependence of the first interlayer spacing for several metal surfaces. For all of the surfaces, the increases in interlayer spacing are essentially linear in temperature below a reduced temperature of 
about 0.55 . The thermal expansion coefficient is proportional to the slope of these curves, and is typically somewhat higher than the bulk value. At higher temperatures, the slope is observed to increase in some cases, and this increase has been described as "anomalous" in some papers. In the case of $\mathrm{Be}(0001)$, the slope is considerably larger than the bulk value even at the lower temperatures. This result was also described as "anomalous." We would like to distinguish between these two "anomalous" effects by describing the larger (but linear) thermal expansion behavior as "enhanced surface expansion" and by describing the nonlinear behavior as "anomalous surface expansion". Because bulk Be has unique phonon spectra and thermal expansion properties, the "enhanced surface expansion" of $\mathrm{Be}(0001)$ could be a consequence of the comparison to its unusual bulk properties [33].

In Table 2 we present a list of all thermal expansion measurements reported in the literature for surfaces. Since all of the studies performed on the temperature dependence of the interlayer distances of surface metals were done using MEIS and LEED, some comments on the differences between MEIS and LEED might improve our understanding of the contrasts between their results. At the elevated temperatures of these measurements, many surfaces will have a significant step density. The intensities at the positions of the diffraction spots in the LEED patterns are primarily the result of the constructive interference of electrons which are scattered from within terraces. The scattering from randomly-placed steps will contribute primarily to the diffuse intensity. Since a LEED I(E) experiment uses intensities only at the positions of the diffraction spots, and since the actual intensities of these spots in the LEED analysis is not nearly as important as the energy of their maxima and minima, the intensities measured in a LEED experiment 
should, in most circumstances, be overwhelmingly dominated by the scattering from terraces, at least if they are wider than the transverse beam coherence length. This will not be true at the limit of extremely rough surfaces, when the diffusely-scattered intensity at the position of the diffraction spots becomes comparable to the sharp diffraction intensity. This precludes the use of normal LEED on surfaces which are very rough, although diffuse LEED might be employed in these conditions. In that situation, we would no longer expect the scattering to be dominated by the atoms on terraces.

In a MEIS experiment, the primary mechanism used to determine the relaxation of surface atoms is the blocking of ions which have been back-scattered from deeper layers. If all surface atoms have the same geometry, a well-defined "blocking dip" is observed in the angular dependence of the scattered ions. In MEIS, there is no "constructive interference" mechanism as there is in diffraction. Therefore there is no enhancement of scattering from terrace atoms over scattering from step atoms. Because of this, a relaxation result obtained from MEIS will have a larger contribution from step atoms than a relaxation result obtained from LEED. This discrepancy should be expected to increase with temperature on surfaces where the thermally-induced step densities are high. In addition, the greater number of layers contributing to the backscattering at higher temperatures results in an increase in the number of visible layers outside the blocking direction. Therefore the blocking pattern at high temperature is less sensitive to the individual relaxations than at lower temperatures. 
While this difference in the measurement techniques cannot explain the lowtemperature discrepancies between the LEED and MEIS results, it might explain why the relaxations measured with MEIS increase dramatically at the higher temperatures on some surfaces. If the surface step density becomes very large, the relaxation measured with MEIS will be much more affected by the relaxation of the step atoms, which could be quite different from the relaxation of the terrace atoms. Two recent studies of the surface thermal expansion of $\mathrm{Ag}(111)$ and $\mathrm{Cu}(111)$ using respectively surface x-ray diffraction [20] and LEED I(E) analysis [20] support this notion. These studies were carried out at temperatures similar to the MEIS studies of these surfaces, and unlike the MEIS results, they did not show any anomalous thermal expansion behavior. This suggests that these two surfaces have quite a different morphology at high temperatures compared to that at low temperatures. The fact that the thermal diffuse scattering dominates the LEED pattern at temperatures above $720 \mathrm{~K}$ may be due to this.

In summary, we have studied the temperature dependence of the top three interlayer spacings of $\operatorname{Ag}(111)$ by low energy electron diffraction. Our results show that between $128 \mathrm{~K}$ and $723 \mathrm{~K}$ the surface expansion behavior is essentially bulk-like with a contraction/expansion transition around $0.3 \mathrm{~T}_{\mathrm{m}}(370 \mathrm{~K})$. At $128 \mathrm{~K}$ the first layer is contracted by $0.5 \%$, which is significantly smaller than the value found by MEIS. The Debye temperature obtained for the first three layers was basically constant and the average values for the first, second and third layers are respectively $165 \pm 8 \mathrm{~K}, 199 \pm 9 \mathrm{~K}$, and 212 $\pm 9 \mathrm{~K}$. Data at temperatures higher than $723 \mathrm{~K}$ could not be obtained in this experiment due to the increase of thermal diffuse scattering. 


\section{Acknowledgment}

We thank Talat Rahman for communicating the $\mathrm{Ag}(111)$ and $\mathrm{Cu}(111)$ experimental results from $\mathrm{P}$. Miceli and $\mathrm{H}$. Over, respectively, prior to their publication. We acknowledge helpful conversations with A. N. Al-Rawi, L. W. Bruch and A. Kader. We also thank W. Moritz for supplying the LEEDFIT code. This research has been supported by NSF grant DMR-9819977 and the Director, Office of Energy Research, Basic Energy

Sciences, Materials Sciences Division, of the U.S. Department of Energy under Contract No. DE-AC03-76SF00098. EAS was supported by CNPq (Brazilian Research Agency). 


\section{References}

[1] P. Statiris, H.C. Lu and T. Gustafsson, Phys. Rev. Lett. 72 (1994) 3574.

[2] J.W.M. Frenken, F. Huussen and J.F. Van der Veen, Phys. Rev. Lett. 58 (1987) 401.

[3] Y. Cao and E. Conrad, Phys. Rev. Lett. 65 (1990) 2808.

[4] E.P. Gusey, H.C. Lu, E. Garfunkel and T. Gustafsson, Surf. Rev. Lett. 3 (1996) 1349.

[5] G. Helgesen, D. Gibbs, A.P. Baddorf, D.M. Zehner and S.G.J. Mochrie, Phys. Rev. B 48 (1993) 15320.

[6] K.H. Chae, H.C. Lu and T. Gustafsson, Phys. Rev. B 54 (1996) 14082.

[7] L.J. Lewis, Phys. Rev. B 50 (1994) 17693.

[8] S. Narasimhan and M. Scheffler, Z. Phys. Chem. 202 (1997) 253.

[9] A. Kara, P. Staikov, A.N. Al-Rawi and T.S. Rahman, Phys. Rev. B 55 (1997) R13440.

[10] J. Xie, S. de Gironcoli, S. Baroni and M. Scheffler, Phys. Rev. B 59 (1999) 970.

[11] M. Schwarz, C. Mayer, P. Von Blanckenhagen and W. Schommers, Surf. Rev. Lett. 4 (1997) 1095.

[12] R. Zivieri, G. Santoro and V. Bortolani, Phys. Rev. B 59 (1999) 15959.

[13] K. Pohl, J.-H. Cho, K. Terakura, M. Scheffler and E.W. Plummer, Phys. Rev. Lett. 80 (1998) 2853.

[14] N. Materer, U. Starke, A. Barbieri, M.A. Van Hove, G.A. Somorjai, G.-J. Kroes and C. Minot, Surf. Sci. 381 (1997) 190.

[15] N. Materer, U. Starke, A. Barbieri, M.A. Van Hove, G.A. Somorjai, G.-J. Kroes and C. Minot, J. Phys. Chem. 99 (1995) 6267.

[16] J. Braun, A. Glebor, A.P. Graham, A. Menzel and J.P. Toennies, Phys. Rev. Lett. 80 (1998) 2638.

[17] N.M. Harrison, X.-G. Wang, J. Muscat and M. Scheffler, private communication.

[18] C.F. Walters, K.F. McCarty, E.A. Soares and M.A. Van Hove, private communication. [19] A.N. Al-Rawi, A. Kara and T.S. Rahman, Surf. Sci. 446 (2000) 17.

[20] T.S. Rahman, private communication.

[21] G. Leatherman and R.D. Diehl, Phys. Rev. B 53 (1996) 4939. 
[22] G. Leatherman, R.D. Diehl, P. Kaukasoina and M. Lindroos, Phys. Rev. B 53 (1995) 10254.

[23] E.A. Soares, V.B. Nascimento, V.E. de Carvalho, C.M.C. de Castilho, A.V. de Carvalho, R. Toomes and D.P. Woodruff, Surf. Sci. 419 (1999) 89.

[24] G. Kleinle, W. Moritz, D.L. Adams and G. Ertl, Surf. Sci. Lett. 219 (1989) L637.

[25] G. Kleinle, W. Moritz and G. Ertl, Surf. Sci. 238 (1989) 119.

[26] H. Over, U. Ketterl, W. Moritz and G. Ertl, Phys. Rev. B 46 (1992) 15438.

[27] H. Over, W. Moritz and G. Ertl, Phys. Rev. Lett. 70 (1993) 315.

[28] W. Moritz and J. Landskron, Surf. Sci. 337 (1995) 278.

[29] A. Barbieri and M.A. Van Hove, http://electron.lbl.gov/leedpack/leedpack.html.

[30] C. Kittel, Introduction to Solid State Physics (Wiley, New York, 1986).

[31] J.B. Pendry, J. Phys. C: Solid Sate 13 (1980) 937.

[32] N.W. Ashcroft and N.D. Mermin, Solid State Physics (Holt-Saunders, Philadelphia, 1976).

[33] E.W. Plummer, private communication. 


\section{Figure Captions}

1. $\operatorname{Ag}(111)$ experimental (thin lines) and theoretical (thick lines) $I(E)$ curves for all temperatures with respective $\mathrm{R}_{\mathrm{P}}$ factor values. For each beam, the temperature increases from the bottom to the top (128K, $243 \mathrm{~K}, 323 \mathrm{~K}, 408 \mathrm{~K}, 513 \mathrm{~K}, 573 \mathrm{~K}, 663 \mathrm{~K}$ and $723 \mathrm{~K})$.

2. (a) Interlayer spacing as a function of temperature for the first three interlayer spacings. (b) Debye temperature as a function of temperature for the first three layers of $\operatorname{Ag}(111)$. A representative error bar is shown.

3. First-layer relaxations for $\operatorname{Ag}(111)$ measured by LEED [this work] and MEIS [1] and calculated using EAM-MD [9] and DFT-QHA [10]. The relaxation is specified as the percentage relaxation relative to the bulk value. The melting temperature $\left(T_{m}\right)$ of $A g$ is $1234 \mathrm{~K}[31]$.

4. Experimental determinations of the first-layer relaxations of several close-packed surfaces, as a function of $\mathrm{T} / \mathrm{T}_{\mathrm{m}}$. 


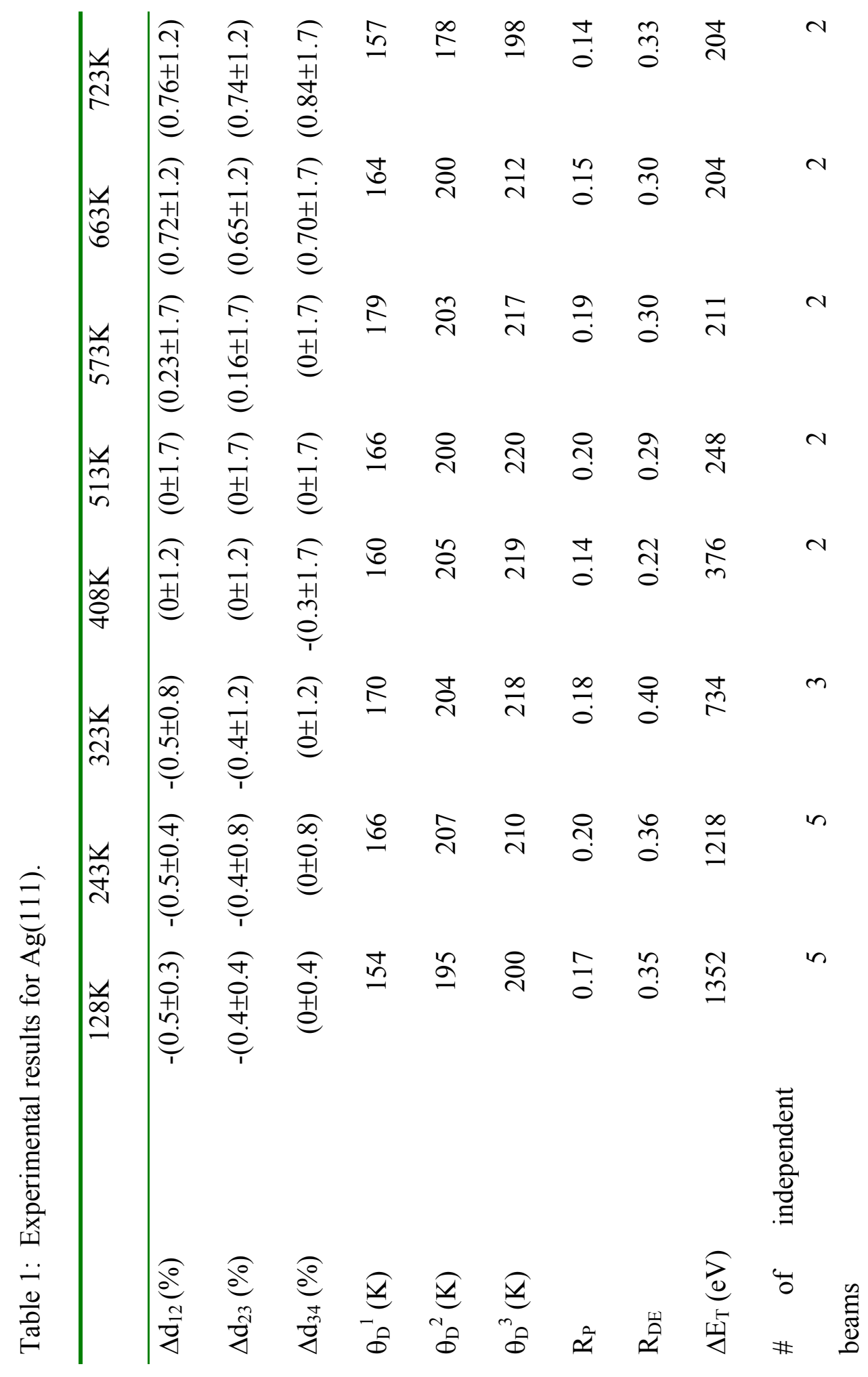


Table 2: List of experimental thermal expansion data for surfaces. $\mathrm{T}_{\mathrm{m}}$ is the bulk melting point temperature. The bulk values were extracted from http://www.webelements.com/

\begin{tabular}{|c|c|c|c|}
\hline \multirow{2}{*}{ Surface } & \multicolumn{2}{|l|}{ thermal expansion coefficients } & \multirow{2}{*}{ Technique } \\
\hline & Surface & Bulk & \\
\hline $\operatorname{Ag}(111)$ & $\begin{array}{l}22.0 \times 10^{-6} \mathrm{~K}^{-1} \text { between } 0.10 \text { and } 0.60 \\
\mathrm{~T}_{\mathrm{m}}\end{array}$ & $18.9 \times 10^{-6} \mathrm{~K}^{-1}$ & LEED [this work] \\
\hline $\operatorname{Ag}(111)$ & $\begin{array}{l}\text { Below } 0.49 \mathrm{~T}_{\mathrm{m}} \text { : similar to the bulk } \\
\text { value } \\
\text { Above } 0.49 \mathrm{~T}_{\mathrm{m}} \text { : increase dramatically } \\
\text { and at } 0.93 \mathrm{~T}_{\mathrm{m}} \text { becomes } 11 \text { times the } \\
\text { bulk value. }\end{array}$ & $18.9 \times 10^{-6} \mathrm{~K}^{-1}$ & MEIS [6] \\
\hline $\mathrm{Cu}(111)$ & $\begin{array}{l}\text { Below } 0.49 \mathrm{~T}_{\mathrm{m}} \text { : similar to the bulk } \\
\text { value } \\
\text { Above } 0.49 \mathrm{~T}_{\mathrm{m}} \text { : increase dramatically } \\
\text { and at } 0.87 \mathrm{~T}_{\mathrm{m}} \text { becomes } 6 \text { times the bulk } \\
\text { value }\end{array}$ & $16.5 \times 10^{-6} \mathrm{~K}^{-1}$ & MEIS [6] \\
\hline $\mathrm{Ni}(111)$ & Bulk-like between $0.23 \mathrm{~T}_{\mathrm{m}}$ and $0.58 \mathrm{~T}_{\mathrm{m}}$ & $13.4 \times 10^{-6} \mathrm{~K}^{-1}$ & MEIS [4] \\
\hline $\operatorname{Be}(0001)$ & $70.0 \times 10^{-6} \mathrm{~K}^{-1}$ between 0.06 and $0.42 \mathrm{~T}_{\mathrm{m}}$ & $11.3 \times 10^{-6} \mathrm{~K}^{-1}$ & LEED [13] \\
\hline $\mathrm{Pb}(110)$ & $\begin{array}{l}\text { Below } 0.50 \mathrm{~T}_{\mathrm{m}}: \text { similar to the bulk } \\
\text { value } \\
\text { Above } 0.50 \mathrm{~T}_{\mathrm{m}}: 3.5 \text { to } 12 \text { times higher }\end{array}$ & $28.9 \times 10^{-6} \mathrm{~K}^{-1}$ & MEIS [2]] \\
\hline $\mathrm{Ni}(001)$ & $\begin{array}{l}\text { Below } 0.52 \mathrm{~T}_{\mathrm{m}} \text { : similar to the bulk } \\
\text { value } \\
\text { Above } 0.52 \mathrm{~T}_{\mathrm{m}} \text { : start to increase } \\
\text { dramatically and at } 0.78 \mathrm{~T}_{\mathrm{m}} \text { is about } 20 \\
\text { times higher than the bulk }\end{array}$ & $13.4 \times 10^{-6} \mathrm{~K}^{-1}$ & $\begin{array}{l}\text { High q resolution } \\
\text { LEED [3] }\end{array}$ \\
\hline
\end{tabular}

